\title{
Implementation science: point-of-care diagnostics in HIV and tuberculosis
}

\author{
Authors: Ankur Gupta-Wright ${ }^{A, B}$ and Yukari C Manabe ${ }^{C}$
}

\begin{abstract}
Implementation science uses methods to promote the scaling up and use of evidence-based practices by health systems to improve quality and outcomes. Its use is vital to maximise the efficiency of limited resources for health care in tropical settings. HIV and tuberculosis (TB) are two of the major causes of morbidity and mortality in sub-Saharan Africa, and globally. Although effective treatments are widely available, lack of diagnosis remains a large barrier to accessing treatment, particularly in resource-limited settings. We explore HIV and TB diagnostics that can be used at point-ofcare in any settings, and outline some important principles and applications of implementation science to aid their application and use. Despite robust evidence of diagnostic accuracy and efficacy in improving patient-centred outcomes, such interventions cannot be fully utilised without addressing operational barriers and knowledge gaps.
\end{abstract}

\section{Introduction}

The research-to-practice gap remains extensive, with the average time taken for evidence-based interventions to reach routine practice being years to decades. ${ }^{1}$ This gap is a particular problem in tropical settings where research findings can often contribute to effective health care, impact public health and deliver more efficient use of limited resources, but many factors inhibit uptake and implementation. This article outlines the importance of implementation science, using examples of point of care diagnostics in HIV and tuberculosis (TB).

\section{What is implementation science?}

Implementation science is the study of methods which promote the uptake of evidence-based practices and findings of research into routine practice, with the aim of improving the quality and effectiveness of health care. ${ }^{2}$ Its focus is often at organisation or policy level, thus requiring a multi-disciplinary approach. ${ }^{3}$ It differs

Authors: ${ }^{\text {A }}$ linical lecturer, Department of Clinical Research, Faculty of Infectious and Tropical Diseases, London School of Hygiene \& Tropical Medicine, London, UK; ${ }^{\text {B }}$ specialty registrar, Department of Infection, St Thomas' Hospital, London, UK; ' professor, Division of Infectious Diseases, Department of Medicine Johns Hopkins School of Medicine, Baltimore, USA from clinical research in that it focusses on rates and quality of use of an intervention rather than the impact of the intervention itself on health outcomes, although implementation studies often also measure 'real-world' effectiveness of interventions.

\section{Challenge of diagnostics in resource-limited settings}

Diagnostics have improved substantially over recent decades, and are a fundamental part of medicine and health care in highincome countries. Due to expensive equipment which requires skilled technicians, reliable electricity and reagent supplies, and stable operating environments, most laboratory tests are inaccessible to patients and clinicians in resource-limited settings. ${ }^{4}$

\section{Key points}

Implementation science is the study of methods promoting the uptake of evidence-based practices and findings of research into routine practice, with the aim of improving the quality and effectiveness of health care

The ASSURED criteria have been developed to help point-of care diagnostics be used in any setting (eg with poor access to electricity), and reduce barriers to implementation.

Rapid HIV sequential testing algorithms allowed for HIV mass testing and subsequent roll-out of life-saving antiretroviral therapy. Self-testing for HIV can be done using oral fluid, and has potential to access hard to reach populations and reduce testing and linkage to care gaps

Urine lipoarabinomannan lateral flow assays significantly improve tuberculosis (TB) diagnosis and can reduce mortality in HIV-positive patients admitted to hospital both with and without classic symptoms of TB (despite imperfect accuracy).

Improving uptake and scaling up of both of these assays will require addressing knowledge gaps and barriers through implementation research

KEYWORDS: HIV, TB, diagnostics, LAM, implementation science 
Box 1. The ASSURED criteria for point-of-care diagnostics (World Health Organisation)

Affordable ( $<5$ US\$ per test)

Sensitive (ideally $99 \%$ )

Specific (ideally $98 \%$ )

User-friendly (requiring minimal training)

Robust (no cold chain) and rapid ( $<1$ hour: same-day results and same-day care) and require little or no operator calibration, and minimal routine maintenance (eg wiping spills, dusting)

Equipment-free (battery-operated, few moving parts, handheld/ compact)

Deliverable (commercially available and approved).

Often, algorithmic care predominates with little diagnostic certainty. This public health approach results in both over- as well as under-treatment. Thus the need for diagnostic methods that can function without access to centralised laboratories (point-ofcare, POC) remain high on research and policy agendas. ${ }^{5}$ POC tests also have the advantage of providing results rapidly, not relying on patients returning to collect results, and have the potential to improve health care services and outcomes in a patient-centred manner. The development of POC tests has focused on infectious diseases in resource-limited settings, for example HIV and, more recently, TB. The ASSURED criteria have been proposed to promote POC diagnostics that are accessible in all settings (Box 1 ).

\section{Burden of HIV and TB}

HIV and TB are still major causes of morbidity and mortality in tropical settings. By 2017, there were estimated to be 36.7 million people living with HIV (PLHIV) worldwide, including 1.8 million new infections that year. ${ }^{6}$ Although HIV is a global epidemic, the burden of prevalence, incidence and mortality lies in sub-Saharan Africa, where $70 \%$ of PLHIV and $65 \%$ of HIV deaths occur. ${ }^{6}$

TB is still the leading single infectious cause of death and one of the top ten leading overall causes of death worldwide. ${ }^{7}$ An estimated 10.4 million people had TB disease in 2016, of whom $25 \%$ lived in the African region. Furthermore, sub-Saharan Africa is where the HIV-associated TB epidemic resides, with an estimated 1 million cases and 0.4 million TB deaths among PLHIV. ${ }^{7}$ Both HIV and TB epidemics are fuelled by underdiagnosis, late presentation and ongoing transmission which cannot be addressed without POC diagnostics.

\section{Point-of-care diagnostics: oral self-testing for HIV}

Antiretroviral therapy (ART) is a potent tool for both individual and public health control of HIV - there were an estimated 21.7 million people receiving ART as of $2017 .{ }^{6,8}$ However, ART requires knowledge of HIV status, and this would not be possible without rapid POC tests for HIV diagnosis, given the lack of access to quality assured centralised laboratories in high HIV-prevalence countries. POC assays for HIV, involving antigen and antibody detection, are referred to as the first generation of POC assays and are widely implemented in sequential algorithms. ${ }^{9,10}$

Whilst POC HIV diagnostics are a success story, under-diagnosis is still responsible for gaps in the HIV care cascade, particularly in young adults and men. ${ }^{11}$ One approach to reaching these populations is community-based testing or self-testing for HIV using oral fluid (eg using the OraQuick assay), which has been shown to have high sensitivity and specificity in field testing, even in the hands of 'less-trained' individuals. ${ }^{12}$ Their use has also shown good subsequent linkage to care and ART initiation. ${ }^{13}$

While the diagnostic accuracy of oral self-testing has been well established (94-100\% sensitivity and $99.5-100 \%$ specificity), there are several implementation questions that need addressing in order to use HIV self-testing to contribute to the 90-90-90 Joint United Nations Programme on HIV and AIDS strategy. ${ }^{14}$ These include global availability of the assays; quality and quality assurance (especially when used for self-testing); acceptability to patients; adaptability to high HIV prevalence settings (the assays were initially designed for high-resource settings); strategies to deliver test kits so as to reach those underserved by facilitybased HIV testing; and linking patients to HIV treatment and/or prevention services. Scaling up of self-testing is only possible based on multicountry implementational evidence confirming feasibility, acceptability and deliverability across many populations and delivery systems.

\section{Point-of-care diagnostics for HIV-associated TB}

Diagnostic tools are also the weakest link in the TB care cascade, and a contributor to poor outcomes. HIV-associated TB is particularly challenging to diagnose owing to atypical clinical presentations, and inadequate sensitivity of traditional diagnostics such as sputum smear microscopy and chest radiography in PLHIV. ${ }^{15,16}$ Missed TB cases result in increased morbidity, mortality and on-going transmission within communities. ${ }^{17,18}$ Almost half of TB found at post-mortem from HIV-positive adults in sub Saharan Africa was undiagnosed at the time of death. ${ }^{19}$ The global "gold standard' TB diagnostic, liquid culture, is expensive, requires considerable infrastructure, is slow, not available in peripheral health care facilities close to patients, and still far from 100\% sensitive. If global targets to reduce TB deaths by $95 \%$ and new cases by $90 \%$ by 2035 are to be met, major improvements in diagnostic strategies are amongst the most pressing needs. ${ }^{20}$

Following many decades of neglect, the TB diagnostic pipeline looks more promising in recent years, including progress with POC assays. The Xpert MTB/RIF assay (Cepheid, USA, 'Xpert'), is the first fully automated, real-time nucleic acid amplification technology for rapid detection of TB and rifampicin resistance, and was approved for TB diagnosis for PLHIV in 2010. ${ }^{21}$ Reviews of the impact of Xpert suggested it improves many diagnostic and therapeutic metrics for TB (eg decreased time to diagnosis and treatment, and pre-treatment loss to follow-up). ${ }^{22}$ Although the first generation platform required a consistent electricity supply and personal computer for operation, a single cartridge unit with battery operation capability was recently introduced, making the Xpert closer aligned to POC testing.

Lipoarabinomannan (LAM), a mycobacterial cell wall lipopolysaccharide, has emerged as a TB diagnostic target, and can be detected in the urine of TB patients using a low cost, POC lateral flow assay (Determine TB-LAM Ag assay, Alere, USA). This truly POC bedside test can be performed with limited training and uses only $60 \mu \mathrm{L}$ of unprocessed urine, which is applied to the test strip, and the result read after 25 minutes. Although the sensitivity of this assay in general populations is suboptimal, diagnostic accuracy is improved in PLHIV, and observational data shows good 
specificity and significant incremental yield in patients with low CD4 cell counts (sensitivity $40-70 \%$ ). ${ }^{23,24}$ Urine LAM detection is thought to be a marker for haematogenously disseminated renal $\mathrm{TB}$, which also explains its association with poor outcomes. ${ }^{25}$

Despite randomised evidence from two randomised clinical trials $^{26,27}$ of significant improvements in patient-centred outcomes among hospitalised PLHIV-increased TB diagnosis and treatment, and mortality reductions in patients with advanced immunosuppression-urine LAM testing has been poorly implemented in African hospitals. ${ }^{28}$

\section{Barriers to implementation of urine LAM testing}

There are several barriers to implementation of urine LAM testing that need to be addressed. The assay does not show impact in all populations, and current conditional World Health Organisation guidance on urine LAM use is difficult to interpret. ${ }^{29}$ The test is recommended for patients with low CD4 cell counts, but CD4 testing is often not available. Although the assay itself is relatively inexpensive (approximately US $\$ 3$ per test), the affordability of implementation by governments with very low per capita health expenditure is unclear, especially when the assay has not (thus far) been supported by international donors. ${ }^{30}$ Supply chains, and optimal methods of testing have not been established for LAM assays (eg bedside testing by clinicians compared to testing in hospital laboratories). It is not clear how LAM should fit into algorithms with other TB diagnostics. Implementation research, ideally performed alongside scale-up and collecting data on implementation strategies and health impacts, are urgently needed to maximise the best TB diagnostic tools we have, such as urine LAM. The knowledge generated will also expedite implementation of better, more sensitive urine LAM assays in the diagnostic pipeline. ${ }^{31}$

\section{Conclusions}

Implementation science is crucial to the successful scaling up and implementation of evidence-based interventions to effect populations and health care en masse. POC diagnostics, for example oral self-tests for HIV and urine LAM assays for HIVassociated TB, are crucial to the control of HIV and TB which remain two of the major public health issues in sub-Saharan Africa. Despite robust evidence of diagnostic accuracy and efficacy in improving patient-centred outcomes, such interventions cannot be fully utilised without addressing operational barriers and knowledge gaps. Diagnostics are only as good as how they are used (innovation without access is not innovation), and 'imperfect' diagnostics used in the right populations can still make a contribution.

\section{References}

1 Morris ZS, Wooding S, Grant J. The answer is 17 years, what is the question: understanding time lags in translational research. $] R$ Soc Med 2011;104:510-20.

2 Eccles MP, Mittman BS. Welcome to Implementation Science. Implement Sci 2006;1:1.

3 Bauer MS, Damschroder L, Hagedorn H, Smith J, Kilbourne AM. An introduction to implementation science for the non-specialist. $B M C$ Psychol 2015;3:1-12.

4 Mabey D, Peeling RW, Ustianowski A, Perkins MD. Diagnostics for the developing world. Nat Rev Microbiol 2004;2:231-40.
5 World Health Organization. Diagnostics for tuberculosis: Global demand and market potential. Geneva: WHO, 2006.

6 Joint United Nations Programme on HIV and AIDS. 2017 Global HIV Statistics. UNAIDS, 2018.

7 World Health Organization. Global tuberculosis report 2017. Geneva: WHO, 2017

8 Johnson LF, May MT, Dorrington RE et al. Estimating the impact of antiretroviral treatment on adult mortality trends in South Africa: A mathematical modelling study. Suthar AB, editor. PLOS Med 2017;14:e1002468

9 Jani IV, Peter TF. How point-of-care testing could drive innovation in global health. N Engl J Med 2013;368:2319-24.

10 Mayhood MK, Afwamba IA, Odhiambo CO et al. Validation, Performance under Field Conditions, and Cost-Effectiveness of Capillus HIV-1/HIV-2 and Determine HIV-1/2 Rapid Human Immunodeficiency Virus Antibody Assays Using Sequential and Parallel Testing Algorithms in Tanzania. J Clin Microbiol 2008;46:3946-51.

11 Napierala Mavedzenge S, Baggaley R, Corbett EL. A review of selftesting for HIV: research and policy priorities in a new era of HIV prevention. Clin Infect Dis 2013;57:126-38.

12 Zachary D, Mwenge L, Muyoyeta $M$ et al. Field comparison of OraQuick ${ }^{\circ}$ ADVANCE Rapid HIV-1/2 antibody test and two blood-based rapid HIV antibody tests in Zambia. BMC Infect Dis 2012;12:183.

13 Sharma M, Ying R, Tarr G, Barnabas R. Systematic review and metaanalysis of community and facility-based HIV testing to address linkage to care gaps in sub-Saharan Africa. Nature 2015;528:S7785.

14 Indravudh PP, Choko AT, Corbett EL. Scaling up HIV self-testing in sub-Saharan Africa. Curr Opin Infect Dis 2018;31:14-24.

15 Monkongdee P, McCarthy KD, Cain KP, Tasaneeyapan T, Nguyen HD, Nguyen TNL et al. Yield of acid-fast smear and mycobacterial culture for tuberculosis diagnosis in people with human immunodeficiency virus. Am J Respir Crit Care Med 2009;180:903-8.

16 Kistan J, Laher F, Otwombe K et al. Pulmonary TB: Varying radiological presentations in individuals with HIV in Soweto, South Africa. Trans R Soc Trop Med Hyg 2017;111:132-6.

17 MacPherson P, Houben RM, Glynn JR, Corbett EL, Kranzer K. Pre-treatment loss to follow-up in tuberculosis patients in lowand lower-middle-income countries and high-burden countries: a systematic review and meta-analysis. Bull World Health Organ 2014:92:126-38.

18 Dimairo M, MacPherson P, Bandason T et al. Hill PC (ed). The Risk and timing of tuberculosis diagnosed in smear-negative TB suspects: A 12-month cohort Study in Harare, Zimbabwe. PLoS One 2010;5:e11849.

19 Gupta RK, Lucas SB, Fielding KL, Lawn SD. Prevalence of tuberculosis in post-mortem studies of HIV-infected adults and children in resource-limited settings: a systematic review and meta-analysis. AIDS 2015;29:1987-2002.

20 World Health Organization. Report: Consensus meeting on high priority target product profiles, 28-29th April 2014. Geneva: WHO 2014.

21 World Health Organization. Automated real-time nucleic acid amplification technology for rapid and simultaneous detection of tuberculosis and rifampicin resistance: Xpert MTB/RIF system. Policy statement. Geneva: WHO, 2011.

22 Auld AF, Fielding KL, Gupta-Wright A, Lawn SD. Xpert MTB/RIF Why the lack of morbidity and mortality impact in intervention trials? Trans R Soc Trop Med Hyg 2016;110:432-44.

23 Gupta-Wright A, Peters JA, Flach C, Lawn SD. Detection of lipoarabinomannan (LAM) in urine is an independent predictor of mortality risk in patients receiving treatment for HIV-associated tuberculosis in sub-Saharan Africa: a systematic review and meta-analysis. BMC Med 2016;14:53. 
24 Shah M, Hanrahan C, Wang ZY et al. Lateral flow urine lipoarabinomannan assay for detecting active tuberculosis in HIV-positive adults. Cochrane Database Syst Rev 2016;5:CD011420.

25 Lawn SD, Gupta-Wright A. Detection of Lipoarabinomannan (LAM) in urine is indicative of disseminated tuberculosis with renal involvement in patients living with HIV and advanced immunodeficiency: evidence and implications. Trans R Soc Trop Med Hyg 2016;110:180-5.

26 Gupta-Wright A, Corbett EL, van Oosterhout J] et al. Rapid urinebased screening for tuberculosis in HIV-positive patients admitted to hospital in Africa (STAMP): a pragmatic, multicentre, parallel-group, double-blind, randomised controlled trial. Lancet 2018;392:292-301.

27 Peter JG, Zijenah LS, Chanda D et al. Effect on mortality of pointof-care, urine-based lipoarabinomannan testing to guide tuberculosis treatment initiation in HIV-positive hospital inpatients: a pragmatic, parallel-group, multicountry, open-label, randomised controlled trial. Lancet 2016:387:1187-97.

28 Médecins Sans Frontières and Stop TB Partnership. Out of Step 2017 TB policies in 29 countries. A survey of prevention, testing and treatment policies and practices. Geneva: Stop TB and MSF, 2017.
29 World Health Organization. The use of lateral flow urine lipoarabinomannan assay (LF-LAM) for the diagnosis and screening of active tuberculosis in people living with HIV: policy. Geneva: WHO, 2015.

30 Reddy KP, Gupta-Wright A, Fielding K et al. Cost-effectiveness of urine-based tuberculosis screening in hospitalised patients with HIV in Africa. Lancet Glob Heal 2019;7:e200-8.

31 Paris L, Magni R, Zaidi F et al. Urine lipoarabinomannan glycan in HIV-negative patients with pulmonary tuberculosis correlates with disease severity. Sci Transl Med 2017;9:eaal2807.

Address for correspondence: Dr Ankur Gupta-Wright, Department of Clinical Research, Faculty of Infectious and Tropical Diseases, London School of Hygiene \& Tropical Medicine, Keppel Street, London WC1E 7HT, UK.

Email: ankurgw@outlook.com

\section{The RCP Medical Training Initiative (MTI)}

Enabling trusts to recruit RCP-endorsed international medical graduates and foster links with hospitals around the world, while providing overseas trainees with the opportunity to work and train in the UK.

\section{For more information, visit www.rcplondon.ac.uk/mti} oremail MTI@rcplondon.ac.uk
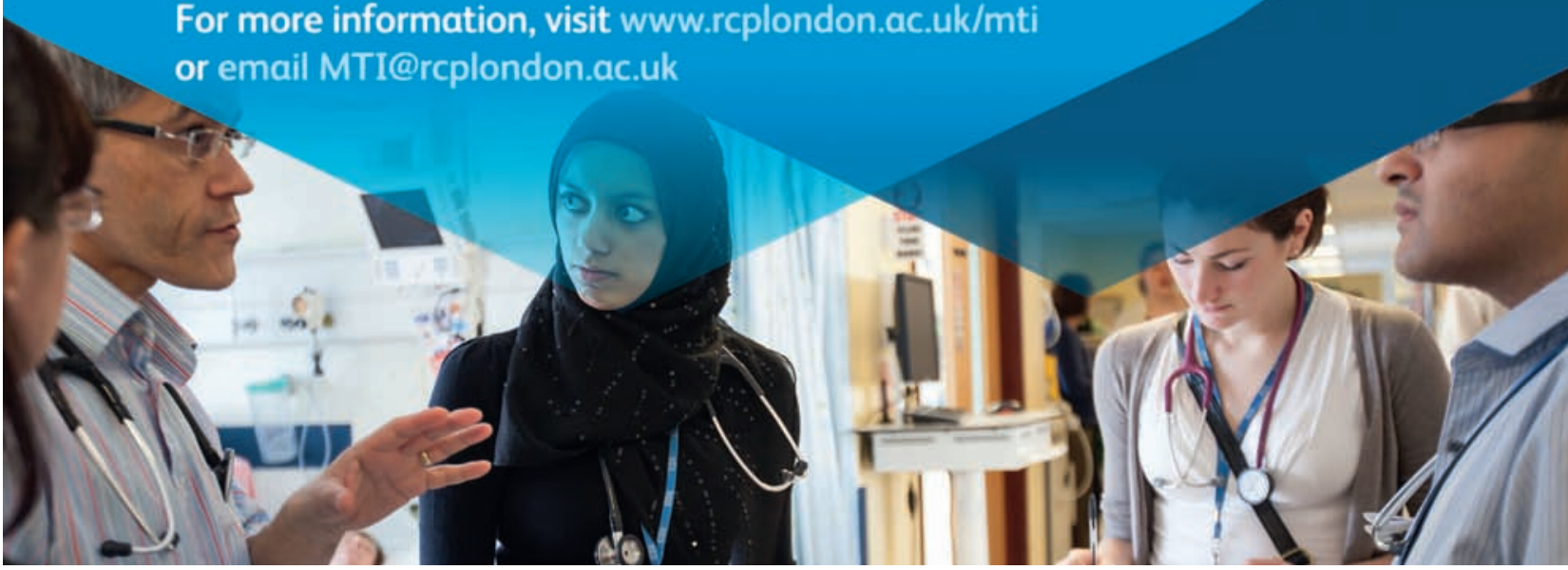